\title{
La sfida dell'ambiente Web 2.0 nella didattica delle lingue minori
}

\author{
Edit Rózsavölgyi ${ }^{1}$
}

\begin{abstract}
Nell'ultimo ventennio lo sviluppo tecnologico, le istanze della crescente globalizzazione e le nuove esigenze della società nei confronti dell'istruzione, in particolare dell'insegnamento delle lingue straniere, oltre che i risultati degli studi sul processo dell'acquisizione nei campi della psicologia cognitiva, della sociologia e della linguistica teorica ed applicata, hanno sollecitato le ricerche nell'ambito della glottodidattica. Gli obiettivi e le modalità dell'apprendimento sono cambiati. Molti lavori hanno evidenziato il fatto che le nuove tecnologie, in particolare gli strumenti dell'ambiente Web 2.0, si prestano ad essere adoperati anche nell'insegnamento delle lingue straniere. Il contesto delle lingue minori offre la possibilità di mettere in atto indagini qualitative nella sfera dell'apprendimento tele-collaborativo contribuendo alle nostre conoscenze in maniera diversa rispetto alle analisi di indirizzo prevalentemente quantitativo che costituiscono la grande maggioranza degli apporti in questo settore di ricerca. In base alle nostre esperienze nell'ambito di un progetto eTandem italo-ungherese, in questo contributo ribadiamo l'utilità di esplorare le specificità individuali degli apprendenti per poter predisporre ambiti di studio sempre più stimolanti e personalizzati nell'ottica di crescere l'autonomia degli studenti.
\end{abstract}

Parole chiave: glottodidattica, eTandem, lingue minori.

\section{Introduzione}

Oggi la comunicazione ha carattere sempre più interculturale, perciò la competenza comunicativa interculturale deve essere sviluppata negli allievi di una L2. Per attuare tale proposito può essere utile adottare nella didattica gli strumenti del Web 2.0 che hanno rivoluzionato il mondo della comunicazione, della condivisione e

1. Università degli Studi di Padova, Italia; edit.r@unipd.it

How to cite this article: Rózsavölgyi, E. (2015). La sfida dell'ambiente Web 2.0 nella didattica delle lingue minori. In F. Helm, L. Bradley, M. Guarda, \& S. Thouësny (Eds), Critical CALL-Proceedings of the 2015 EUROCALL Conference, Padova, Italy (pp. 490-494). Dublin: Research-publishing.net. http://dx.doi.org/10.14705/rpnet.2015.000381 
della collaborazione tra persone geograficamente distanti. Promuovere pratiche telecollaborative favorisce la cooperazione didattica con costi contenuti e con il coinvolgimento di un numero maggiore di studenti in scambi interculturali dove gli ambienti virtuali sostituiscono quelli reali.

La letteratura di riferimento è basata in larga misura su lavori svolti nel contesto della lingua inglese. E'stato riconosciuto però che il panorama socio-culturale può influenzare i risultati delle indagini (Ushioda \& Dörnyei, 2012). Questo contributo si pone l'obiettivo di indagare, in base a una sperimentazione pilota eTandem italo-ungherese, su che cosa di positivo l'uso delle nuove tecnologie nel curriculum universitario può aggiungere alla metodologia dell'insegnamento delle lingue straniere mettendo in evidenza gli aspetti specifici delle lingue minori. Riporteremo i punti di forza e di criticità della nostra esperienza come risultati di una microanalisi intensiva delle interazioni, dei dialoghi collaborativi e delle dinamiche sociali, culturali e psicologiche dei partecipanti al progetto.

\section{Background teorico}

La principale innovazione nella glottodidattica e in particolare nelle ricerche sull'apprendimento di una L2 degli ultimi anni è stata quella di porre al centro dell'attenzione l'apprendente e la sua autonomia. Quest'ultima viene vista come uno degli obiettivi dell'apprendimento stesso. La sua realizzazione condiziona fortemente la pratica didattica e porta inevitabilmente alla trasformazione dei ruoli di ambedue i protagonisti, docenti e studenti, dell'acquisizione linguistica.

Il quadro di riferimento teorico delle nostre ricerche si colloca nell'ambito del cognitivismo. La teoria socio-cognitiva definisce l'apprendimento come acquisizione di conoscenze attraverso l'elaborazione cognitiva di informazioni. Viene enfatizzato il ruolo dell'agentività umana (agency), intesa come la capacità di agire attivamente nel contesto in cui si è inseriti, per fare scelte, per progettare e generare azioni mirate a determinati obiettivi e per motivarne e disciplinarne l'esecuzione. Essa comprende anche la facoltà metacognitiva di contemplare se stessi e di riflettere sulle proprie azioni e sui propri ragionamenti (Bandura, 2006). L'idea di una partecipazione attiva della persona nel proprio processo di apprendimento, sotto forma di autoregolazione (selfregulation), è parte integrante anche dell'approccio socio-culturale contemporaneo di impronta vygotskyana (Dörnyei, 2005, p. 191).

L'agentività/autoregolazione e la personalità sono i concetti chiave di collegamento delle teorie socio-cognitiva e socio-culturale da una parte e di quella dell'autonomia 
dall'altra, le prime rappresentando il punto di partenza dello sviluppo dell'autonomia, la seconda costituendone uno dei risultati più significativi. Non si può prescindere inoltre del ruolo fondamentale che la motivazione gioca nell'innesco dei processi di autonomia e dell'apprendimento in generale. La relazione tra motivazione, autonomia e agentività/autoregolazione è data dal fatto che tutte richiedono il coinvolgimento attivo da parte dell'apprendente.

Nell'ottica delineata sopra l'autonomia significa dunque capacità di avere il controllo sulle proprie azioni, di mantenere e aumentare la propria motivazione di fronte a fattori di distrazione ed esperienze emotivamente negative, di avere la facoltà di riflettere in maniera produttiva sul proprio processo di apprendimento, di mettersi in una posizione di partecipante attivo nell'apprendimento.

\section{L'eTandem italo-ungherese Danubadria}

La sperimentazione che costituisce la base empirica della nostra ricerca è rappresentata dal progetto eTandem chiamato Danubadria in virtù del fatto che coinvolge l'ungherese e l'italiano, due lingue parlate lungo due vie di comunicazione europee importantissime: il Danubio e il Mar Adriatico. Il programma è stato realizzato nel 2011. In questa sede per mancanza di spazio non ci è concesso fornire una descrizione dettagliata della nostra esperienza per la quale si rimanda a Rózsavölgyi e Guglielmi (2011) e Rózsavölgyi (2014). Riporteremo qui i risultati della nostra indagine soffermandoci sugli aspetti positivi di Danubadria che superano di gran lunga le aree di criticità, ridotte di fatto ad alcuni contesti della pratica quotidiana come difficoltà nel trovarsi con scadenza regolare prestabilita per le sessioni eTandem dovuta al calendario accademico differente delle università di provenienza, divario nei tempi di realizzazione dei compiti, mancanza di variazione dei canali di comunicazione e simili.

La nostra ricerca ha evidenziato che la spinta motivazionale iniziale dello studente di ungherese è alta e di tipo integrativo, legata alla sfera affettiva. Abbiamo riscontrato nello stesso tempo una scarsa autostima negli apprendenti e difficoltà nel 'mettersi in gioco'. Questo quadro ci ha dato un'indicazione precisa sul fatto che tra le finalità del progetto doveva essere contemplato il potenziamento dell'agentività degli studenti a pari passo con la capacità comunicativa interculturale. A questo proposito sono stati messi in opera diversi stratagemmi tra i quali elenchiamo qui di seguito i più significativi.

In fase progettuale l'elaborazione dei dati di un questionario compilato dai partecipanti ha reso possibile una formazione 'sapiente' delle coppie. E' stato scelto 
un protocollo con autogestione mediata da docenti-tutor con valenza curricolare per creare un ambiente personalizzato e flessibile. Per garantire una cornice minima di lavoro comune e per regolare le interazioni si è istituito un protocollo di intesa condiviso con gli studenti.

La piattaforma di lavoro (Wiki PBworks con accesso riservato ai partecipanti) è stata percepita come uno spazio diviso in due corsie: la prima è un forum di informazione e di assistenza di carattere generale e uno spazio di socializzazione per l'intero gruppo di eTandem, la seconda uno spazio didattico personalizzato per ogni singola coppia con forum, archiviazione dei materiali e tutoraggio. Si è creato così un doppio livello di scaffolding ('impalcatura'), uno interno specifico di ogni coppia con sostegno tra pari e da parte dei tutor e uno esterno 'perimetrale' ad uso di tutto il gruppo. Questo tipo di costruzione ha sollecitato un alto grado di intensità dialogica e di vicinanza tra tutti i partecipanti, docenti-tutor compresi e ha reso possibile percorsi personalizzati.

Il programma task-based, organizzato seguendo anche i suggerimenti degli apprendenti, ha ridotto la potenziale dispersione delle informazioni grazie alla possibilità di archiviare e condividere i materiali e agli interventi sul forum. Ha richiesto processi di negoziazione e mediazione impegnativi tra i pari e $\mathrm{i}$ tutor rafforzando l'identificazione in un progetto formativo comune, oltre che l'interdipendenza positiva tra i partner e l'efficacia dell'azione attiva di tutoraggio sostenendo alla base le istanze motivazionali e di autonomia.

Nel campo delle competenze linguistiche abbiamo lasciato libertà per ogni coppia di decidere quali aspetti della lingua dovessero essere rafforzati durante la creazione dei materiali di consegna e di trovare un equilibrio tra la correttezza grammaticale e la scioltezza discorsiva privilegiando così obiettivi pragmalinguistici ('imparare a fare con la lingua").

\section{Conclusioni}

I vantaggi della tele-collaborazione integrata e di sostegno all'apprendimento in eTandem Danubadria possono essere ricondotti a due principali filoni di dinamiche formative:

- costruzione collaborativa dei contenuti e del programma di lavoro:

- doppio circuito di tutoraggio personalizzato dove condividere i processi di apprendimento. 
La personalizzazione nell'eTandem ha significato per gli studenti l'adattamento dei propri approcci, bisogni e aspettative all'ambiente tecnologico negoziando con il partner e l'incremento della consapevolezza sul proprio apprendimento e agentività; per i tutor il coinvolgimento cognitivo, sociale ed emotivo degli studenti nel processo dell'apprendimento interpretando e stimolando le loro motivazioni, creando delle sfide potenzialmente raggiungibili, assecondando le caratteristiche specifiche delle singole persone nella strutturazione dei task e stimolando riflessioni metacognitive.

Questo tipo di lavoro 'fino' ha naturalmente ricadute di diversi esiti sulle persone coinvolte, ma può essere realizzato senz'altro con più facilità nell'ambito delle lingue 'di nicchia' che a loro volta possono contribuire con indagini di matrice qualitativa come quella che abbiamo riportato qui agli studi sul CALL in generale.

\section{Riferimenti bibliografici}

Bandura, A. (2006). Toward a psychology of human agency. Perspectives on Psychological Science, 1(2), 164-180. doi:10.1111/j.1745-6916.2006.00011.x

Dörnyei, Z. (2005). The psychology of the language learner: individual differences in second language acquisition. London: Lawrence Erlbaum.

Rózsavölgyi, E. (2014). Motivare gli studenti in ambiente Web 2.0. In C. Cervini \& A. C. Valdiviezo

V. (a cura di), Dispositivi formativi e modalità ibride per l'apprendimento linguistico (p. 16). Bologna: CLUEB, Collana Contesti Linguistici. Retrieved from http://clueb.it/libreria/ contesti-linguistici/dispositivi-formativi-e-modalita-ibride-per-lapprendimento-linguistico/

Rózsavölgyi, E., \& Guglielmi, L. (2011). Peer correction and learner's self perception of motivation and autonomy in an Italian-Hungarian eTandem. In G. Raţă (Ed.), Academic days of Timişoara: language education today (pp. 450-465). Newcastle upon Tyne: Cambridge Scholars Publishing.

Ushioda, E., \& Dörnyei, Z. (2012). Motivation. In S. Gass \& A. Mackey (Eds.), The Routledge handbook of second language acquisition (pp. 396-409). New York: Routledge. doi:10.1057/9781137032829.0009 


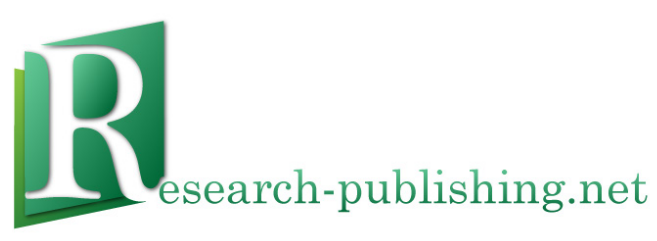

Published by Research-publishing.net, not-for-profit association Dublin, Ireland; info@research-publishing.net

(C) 2015 by Research-publishing.net (collective work)

(C) 2015 by Author (individual work)

Critical CALL - Proceedings of the 2015 EUROCALL Conference, Padova, Italy Edited by Francesca Helm, Linda Bradley, Marta Guarda, and Sylvie Thouësny

Rights: All articles in this collection are published under the Attribution-NonCommercial -NoDerivatives 4.0 International (CC BY-NC-ND 4.0) licence. Under this licence, the contents are freely available online (as PDF files) for anybody to read, download, copy, and redistribute provided that the author(s), editorial team, and publisher are properly cited. Commercial use and derivative works are, however, not permitted.

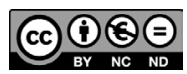

Disclaimer: Research-publishing.net does not take any responsibility for the content of the pages written by the authors of this book. The authors have recognised that the work described was not published before, or that it is not under consideration for publication elsewhere. While the information in this book are believed to be true and accurate on the date of its going to press, neither the editorial team, nor the publisher can accept any legal responsibility for any errors or omissions that may be made. The publisher makes no warranty, expressed or implied, with respect to the material contained herein. While Research-publishing.net is committed to publishing works of integrity, the words are the authors' alone.

Trademark notice: product or corporate names may be trademarks or registered trademarks, and are used only for identification and explanation without intent to infringe.

Copyrighted material: every effort has been made by the editorial team to trace copyright holders and to obtain their permission for the use of copyrighted material in this book. In the event of errors or omissions, please notify the publisher of any corrections that will need to be incorporated in future editions of this book.

Typeset by Research-publishing.net

Fonts used are licensed under a SIL Open Font License

ISBN13: 978-1-908416-28-5 (Paperback - Print on demand, black and white)

Print on demand technology is a high-quality, innovative and ecological printing method; with which the book is never 'out of stock' or 'out of print'.

ISBN13: 978-1-908416-29-2 (Ebook, PDF, colour)

ISBN13: 978-1-908416-30-8 (Ebook, EPUB, colour)

Legal deposit, Ireland: The National Library of Ireland, The Library of Trinity College, The Library of the University of Limerick, The Library of Dublin City University, The Library of NUI Cork, The Library of NUI Maynooth, The Library of University College Dublin, The Library of NUI Galway.

Legal deposit, United Kingdom: The British Library.

British Library Cataloguing-in-Publication Data.

A cataloguing record for this book is available from the British Library.

Legal deposit, France: Bibliothèque Nationale de France - Dépôt légal: décembre 2015. 